\title{
HOMFLY POLYNOMIALS AS VASSILIEV LINK INVARIANTS
}

\author{
TAIZO KANENOBU \\ Department of Mathematics, Osaka City University \\ Sumiyoshi-ku, Osaka 558-8585, Japan \\ E-mail: kanenobu@sci.osaka-cu.ac.jp \\ YASUYUKI MIYAZAWA \\ Department of Mathematics, Yamaguchi University, Yamaguchi 753-8512, Japan \\ E-mail: miyazawa@cc.yamaguchi-u.ac.jp
}

\begin{abstract}
We prove that the number of linearly independent Vassiliev invariants for an $r$-component link of order $n$, which derived from the HOMFLY polynomial, is greater than or equal to $\min \{n,[(n+r-1) / 2]\}$.
\end{abstract}

Introduction. Let $V_{n}$ denote the vector space consisting of all Vassiliev knot invariants of order less than or equal to $n$. There is a filtration

$$
V_{0} \subset V_{1} \subset V_{2} \subset \cdots \subset V_{n} \subset \cdots
$$

in the entire space of Vassiliev knot invariants. Each $V_{n}$ is finite-dimensional. Vassiliev [V] studied for the special cases when $n$ is small: $V_{0}=V_{1}$, which consists of a constant map (Propositions 3 and 5 ), and $V_{2} / V_{1}$ is a one-dimensional vector space, whose basis is the second coefficient of the Conway polynomial. The dimensions for small $n$ are found by using the computer by Bar-Natan and Stanford (cf. [BN; B1, p. 282 ]): For $n=$ $1,2,3,4,5,6,7, \operatorname{dim} V_{n} / V_{n-1}=0,1,1,3,4,9,14$, respectively.

On the other hand, Bar-Natan (cf. $[\mathrm{BN}]$ ) showed that the $n$th coefficient of the Conway polynomial is of order less than or equal to $n$. Birman and Lin [BL] and Gusarov [G] proved that the Jones, HOMFLY, and Kauffman polynomials of a knot can be interpreted as an infinite sequence of Vassiliev knot invariants, and as a corollary they proved that $\operatorname{dim} V_{n} / V_{n-1} \geq 1$ for every $n \geq 2$ using the HOMFLY polynomial [BL, Corollary 4.2 (i)].

1991 Mathematics Subject Classification: Primary 57M25.

Key words and phrases: link, Vassiliev link invariant, HOMFLY polynomial, Jones polynomial, Conway polynomial.

The paper is in final form and no version of it will be published elsewhere. 
Stanford [S1] generalized this for a link. In the special case of the Jones polynomial, the statement is as follows: Let $V_{K}(t)$ be the Jones polynomial of a knot $K$. Set $t=e^{x}$ and expand $e^{x}$ via its Taylor series to obtain a power series expansion of $V_{K}(t)$ :

$$
V_{K}\left(e^{x}\right)=\sum_{n=0}^{\infty} u_{n}(K) x^{n}
$$

Then the coefficient $u_{n}(K)$ of $x^{n}$ is a Vassiliev invariant of order less than or equal to $n$. Melvin and Morton $[\mathrm{MM}]$ have shown that the order is just $n$. From this, we see that the $n$th derivative of $V_{K}(t)$ evaluated at $1, V_{K}^{(n)}(1)$, is a Vassiliev invariant of order $n$. See Theorem 1.

In this paper, we study Vassiliev link invariants derived from the HOMFLY polynomial in a similar form. Let $P_{k}^{(\ell)}(L ; 1)$ be the $\ell$ th derivative of the $k$ th coefficient polynomial of the HOMFLY polynomial of a link $L$ evaluated at 1 . In particular, $P_{k}(L ; 1)=a_{k}(L)$, the $k$ th coefficient of the Conway polynomial. We show that $P_{k}^{(\ell)}(L ; 1)$ is a Vassiliev link invariant of order $\max \{k+\ell, 0\}$; in the following, $P_{k}^{(\ell)}$ indicates this Vassiliev link invariant. Furthermore, we have:

Main Theorem. Let $s=\min \{n,[(n+r-1) / 2]\}$. Then the dimension of the subspace of the Vassiliev invariants for an r-component link of order $n$ spanned by the following Vassiliev invariants is $s$ :

$$
P_{2 i-r+1}^{(n+r-2 i-1)}, \quad i=0,1, \ldots, s .
$$

Here [] denotes the greatest integer function.

Let us restrict attention to knots. This theorem gives a lower bound of the dimension of the HOMFLY subspace of $V_{n} / V_{n-1}$ defined by Birman and Lin [BL, p. 264], where they give the bound for $n \leq 4$. Meng [Me] shows that the dimension of the HOMFLY subspace of $V_{n} / V_{n-1}$ is $[n / 2]$ applying the bracket weight system. Also, Chmutov and Duzhin [CD] show $\operatorname{dim} V_{n} / V_{n-1} \leq(n-1)$ !, and more recently, $\mathrm{Ng}[\mathrm{N}]$ shows $\operatorname{dim} V_{n} / V_{n-1} \leq(n-2) ! / 2$ if $n \geq 6$.

This paper consists of seven sections. In Sect. 1, we define a Vassiliev link invariant and give some properties following Birman and Lin [BL], Birman [B1, B2] and Stanford [S1]. In Sect. 2, we show that $P_{k}^{(\ell)}$ is a Vassiliev link invariant of order $\max \{k+\ell, 0\}$ (Lemma 1). From the proof of this, we get a useful recursion formula (2.7) for calculating the $P_{k}^{(\ell)}$-value of the $(k+\ell)$-configuration. Using this formula, we calculate a family of configurations (Lemma 2), which is a key step for proving our main result. In Sect. 3, we give some results analogous to those in Sect. 2 for the Jones polynomial. It is known that Vassiliev knot invariants form an algebra, which means that the product of a Vassiliev invariant of order $\leq p$ and one of $\leq q$ is a Vassiliev invariant of order $\leq p+q$, which is shown by Lin (unpublished) and Bar-Natan [BN]. In Sect. 4, we prove this for a link (Theorem 2), and also give a formula for calculating the value of the product of Vassiliev invariants for a $(p+q)$-configuration (Proposition 9). In Sect. 5, we give a basis for the space $V_{4}$ in terms of the invariants derived from the HOMFLY polynomial by making use of the result of Birman and Lin [BL]. Using this we get various relations among polynomial invariants regarding them as Vassiliev invariants of small order. In Sect. 6, 
we give a relation among $P_{2 i}^{(n-2 i)}$ 's (Theorem 3), which is obtained by generalizing some formulas given in Sect. 5. This theorem, together with Lemma 2 in Sect. 2, implies Main Theorem for a knot (Theorem 4). In Sect. 7, we generalize Theorem 4 to a link (Theorem $5)$, thereby completing the proof of Main Theorem.

Acknowledgements. The authors would linke to thank Hirozumi Fujii, who helped them in drawing Fig. 10.

1. Vassiliev link invariants. An $r$-component link is the image of oriented $r$ circles under an embedding into an oriented 3-sphere $S^{3}$. A knot is a 1-component link. An $r$-component link is trivial if it is planar, which we denote by $U^{r} ; U^{1}=U$, which is a trivial knot, and $U^{0}=\emptyset$.

An $r$-component singular link is the image of oriented $r$ circles under an immersion into $S^{3}$ whose only singularities are transverse double points. We assume that a double point on a singular link is a rigid (or flat) vertex, which means that there is a neighborhood around each double point in which the singular link is contained in a plane. Two $r$ component singular links with $n$ double points are equivalent if there is an isotopy of $S^{3}$ which takes one to the other and which preserves the orientation of each component and the rigidity of each double point. This equivalence relation is called rigid vertex isotopy.

Let $v$ be an isotopy invariant of an $r$-component link, which takes values in the rational numbers $\mathbb{Q}$. Then $v$ can be uniquely extended to an $r$-component singular link invariant by the Vassiliev skein relation:

$$
v\left(L_{\times}\right)=v\left(L_{+}\right)-v\left(L_{-}\right)
$$

where $L_{\times}$is a singular link with $x$ a double point and $L_{+}, L_{-}$are ones obtained from $L_{\times}$by replacing $x$ by a positive crossing and a negative crossing, respectively; see Fig. 1. Let $L^{n}=L_{x_{1}, x_{2}, \ldots, x_{n}}$ be a singular link with $n$ double points $x_{1}, x_{2}, \ldots, x_{n}$, and $L_{x_{1}\left(\epsilon_{1}\right), x_{2}\left(\epsilon_{2}\right), \ldots, x_{n}\left(\epsilon_{n}\right)}$ be a non-singular link obtained from $L^{n}$ by replacing each double point $x_{i}$ by a positive crossing $x_{i}(+)$ or a negative crossing $x_{i}(-)$. We see that $v\left(L^{n}\right)$ is a linear combination of the $v$-values of $2^{n}$ links:

$$
v\left(L^{n}\right)=\sum_{\epsilon_{i}= \pm}(-1)^{\mu(\epsilon)} v\left(L_{x_{1}\left(\epsilon_{1}\right), x_{2}\left(\epsilon_{2}\right), \ldots, x_{n}\left(\epsilon_{n}\right)}\right),
$$

where $\mu(\epsilon)$ is the number of minus signs in $\epsilon_{1}, \epsilon_{2}, \ldots, \epsilon_{n}$; cf. [B2, (2)].

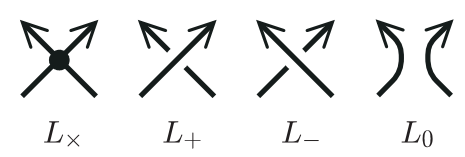

Fig. 1

We call $v$ a Vassiliev (finite-type) link invariant if it satisfies the following axiom:

(1.3) There exists an integer $n$ such that $v(L)=0$ for any singular link $L$ with more than $n$ double points.

The smallest such an integer $n$ is the order of $v$. In the special case of a knot, this reduces to Vassiliev's knot invariant. Stanford [S1] introduces one more axiom in order to relate 
the values of $v$ on links with different number of components, which we do not adopt in this paper.

The following is an immediate consequence of (1.1).

Proposition 1. The value of a Vassiliev invariant of a singular link shown in Fig. 2 is zero.

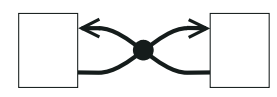

Fig. 2

The $n$-configuration which an $r$-component singular link with $n$ double points respects is the $n$ pairs of points on oriented $r$ circles; cf. [BL, p. 240; B1, p. 273; B2, p. 4]. We use a chord-diagram of order $n$ to represent it, that is, oriented $r$ circles with $n$ chords joining the paired points as in Figs. 4-6. We shall not distinguish strictly a chord-diagram from a configuration.

The following is due to Stanford [S1, Proposition 1.1]; cf. [B1, Lemma 1; B2, Proposition 1].

Proposition 2. Two r-component singular links with $n$ double points become equivalent after an appropriate series of crossing changes if and only if they respect the same $n$-configuration.

In particular, any $r$-component link becomes trivial after an appropriate series of crossing changes. Thus we have

Proposition 3. A Vassiliev link invariant of order 0 is a locally constant map (i.e. it depends only on the number of components).

The singular link shown in Fig. 2 respects the configuration given in Fig. 3, which we call inadmissible. A configuration is called admissible if it is not inadmissible. Thus for any inadmissible configuration, there is a singular link respecting it whose value of any Vassiliev link invariant is zero. (For a singular knot, such an immersion is called a good model in [BL, p. 242].)

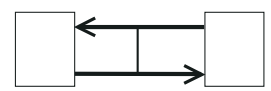

Fig. 3

Now we consider calculating a Vassiliev link invariant of a singular link with fixed number of components. Let us suppose that we have made a list of the distinct admissible $j$-configurations $\alpha_{i}^{j} ; 1 \leq i \leq s_{j}, j=1,2, \ldots$, and chosen, for each $\alpha_{i}^{j}$, a singular link $M_{i}^{j}$ respecting it. By Proposition 2, using a resolution tree, the value of a Vassiliev link invariant of a singular link is given as follows (cf. [LM, Proof of Theorem 2.4; B2, Proposition 2]):

Proposition 4. Let $v$ be a Vassiliev link invariant of order $\leq m$, and $L^{n}$ a singular link respecting the admissible $n$-configuration $\alpha_{p}^{n}, n \leq m$. Then

$$
v\left(L^{n}\right) \equiv v\left(M_{p}^{n}\right)
$$


where "三" denotes equality up to a $\mathbb{Z}$-linear combination of $v\left(M_{i}^{j}\right), 1 \leq i \leq r_{j}, n+1 \leq$ $j \leq m$. In particular, if $m=n$, then "三” is " $=$ ", and so the $v$-value of a singular link with $n$ double points depends only on its configuration.

Let $v$ be a Vassiliev invariant of order $\leq n$, and $\alpha^{n}$ an $n$-configuration. Then by virtue of this proposition, we define $v\left(\alpha^{n}\right)$ by the $v$-value of any singular link respecting $\alpha^{n}$.

Since any 1-configuration is inadmissible, we have (cf. [CD, Examples 1.2.2 and 1.2.3]):

Proposition 5. A Vassiliev knot invariant of order $\leq 1$ is a constant map; $V_{0}=V_{1}$.

There are linear relations among the $v$-values of singular links. It is known $[\mathrm{V}, \mathrm{S} 1$, $\mathrm{BN}$ ] that the finite set of 4-term relations suffice to determine a Vassiliev link invariant of order $m$. Thus we can find a consistent set of rational numbers $\left\{v\left(M_{i}^{j}\right) \mid 1 \leq i \leq s_{j}, j=\right.$ $1,2, \ldots, m\}$ such that we can determine an invariant; this assignment is called an actuality table for a Vassiliev link invariant. The method for making an actuality table for a knot is explained in [BL, B2].

2. The HOMFLY polynomial. The HOMFLY polynomial $P(L ; t, z) \in \mathbb{Z}\left[t^{ \pm 1}, z^{ \pm 1}\right]$ [FYHLMO, PT] is an invariant of a link $L$, which is defined, as in $[\mathrm{J}]$, by the following formulas:

$$
\begin{gathered}
P(U ; t, z)=1 \\
t^{-1} P\left(L_{+} ; t, z\right)-t P\left(L_{-} ; t, z\right)=z P\left(L_{0} ; t, z\right),
\end{gathered}
$$

where $L_{+}, L_{-}, L_{0}$ are three links that are identical except near one point where they are as in Fig. $1 ; L_{+}$is obtained from $L_{-}$by changing the crossing, and $L_{0}$ is obtained by smoothing the crossing.

By [LM, Proposition 22], the HOMFLY polynomial of an $r$-component link $L=$ $K_{1} \cup K_{2} \cup \ldots \cup K_{r}$ is of the form

$$
P(L ; t, z)=\sum_{i=1}^{N} P_{2 i-1-r}(L ; t) z^{2 i-1-r},
$$

where $P_{2 i-1-r}(L ; t) \in \mathbb{Z}\left[t^{ \pm 1}\right]$ is called the $(2 i-1-r)$ th coefficient polynomial of $P(L ; t, z)$ and the powers of $t$ which appear in it are either all even or odd, depending on whether $r$ is odd or even. Let $P_{k}^{(\ell)}(L ; t)$ be the $\ell$ th derivative of $P_{k}(L ; t)$. Note that $P_{k}^{(\ell)}(L ;-t)=$ $(-1)^{k+\ell} P_{k}^{(\ell)}(L ; t)$. By [Kw, Lemma 1.7], if $1 \leq i \leq r-1$, then $P_{2 i-1-r}(L ; t)$ is divisible by $\left(t^{-1}-t\right)^{r-i}$. In particular, by [LM, Proposition 22],

$$
P_{1-r}(L ; t)=t^{2 \lambda}\left(t^{-1}-t\right)^{r-1} \prod_{j=1}^{r} P_{0}\left(K_{j} ; t\right),
$$

where $\lambda$ is the total linking number of $L$ defined by $\lambda=\sum_{i<j} l k\left(K_{i}, K_{j}\right)$, and for a knot $K, P_{0}(K ; 1)=1$. Thus we have

Proposition 6. If $L$ is an $r$-component link, $r \geq 2$, then

$$
P_{2 i-1-r}^{\left(m_{i}\right)}(L ; 1)= \begin{cases}(r-1) !(-2)^{r-1} & \text { if } i=1, m_{1}=r-1 ; \\ 0 & \text { if } 1 \leq i \leq r-1,0 \leq m_{i} \leq r-i-1 .\end{cases}
$$


Lemma 1. $P_{k}^{(\ell)}(L ; 1)$ is a Vassiliev link invariant of order less than or equal to $\max \{k+\ell, 0\}$.

Proof. First we prepare the formula (2.5) below. The equation (2.1b) implies

$$
P_{k}\left(L_{+} ; t\right)-P_{k}\left(L_{-} ; t\right)=\left(t^{2}-1\right) P_{k}\left(L_{-} ; t\right)+t P_{k-1}\left(L_{0} ; t\right) .
$$

Differentiating the both sides $\ell$ times, we obtain

$$
\begin{aligned}
P_{k}^{(\ell)}\left(L_{+} ; t\right)-P_{k}^{(\ell)}\left(L_{-} ; t\right) & \\
= & \left(t^{2}-1\right) P_{k}^{(\ell)}\left(L_{-} ; t\right)+2 \ell t P_{k}^{(\ell-1)}\left(L_{-} ; t\right)+\ell(\ell-1) P_{k}^{(\ell-2)}\left(L_{-} ; t\right) \\
& \quad+t P_{k-1}^{(\ell)}\left(L_{0} ; t\right)+\ell P_{k-1}^{(\ell-1)}\left(L_{0} ; t\right) .
\end{aligned}
$$

Substituting $t=1$, this becomes

$$
\begin{aligned}
& P_{k}^{(\ell)}\left(L_{+} ; 1\right)-P_{k}^{(\ell)}\left(L_{-} ; 1\right) \\
& \quad=2 \ell P_{k}^{(\ell-1)}\left(L_{-} ; 1\right)+\ell(\ell-1) P_{k}^{(\ell-2)}\left(L_{-} ; 1\right)+P_{k-1}^{(\ell)}\left(L_{0} ; 1\right)+\ell P_{k-1}^{(\ell-1)}\left(L_{0} ; 1\right) .
\end{aligned}
$$

We use induction on $k+\ell$. If $k+\ell \leq 0$, then the lemma follows from Prosposition 5 . Suppose that the lemma is true for $k+\ell<n$. Let $L_{\times}^{n+1}$ be a singular link with $n+1$ double points $x_{1}, x_{2}, \ldots, x_{n}, x_{n+1}$, and $L_{+}^{n}, L_{-}^{n}, L_{0}^{n}$ be three singular links with $n$ double points obtained from $L_{\times}^{n+1} ; L_{+}^{n}$ and $L_{-}^{n}$ by changing $x_{n+1}$ to a positive crossing $x_{n+1}(+)$ and a negative crossing $x_{n+1}(-)$, respectively, and $L_{0}^{n}$ by smoothing $x_{n+1}$.

From (1.2), we have

$$
\begin{aligned}
P_{k}^{(\ell)}\left(L_{\times}^{n+1} ; 1\right) & =\sum_{\epsilon=\left(\epsilon_{1}, \ldots, \epsilon_{n}, \epsilon_{n+1}\right)}(-1)^{\mu(\epsilon)} P_{k}^{(\ell)}\left(L_{x, x_{n+1}\left(\epsilon_{n+1}\right)} ; 1\right) \\
& =\sum_{\epsilon^{\prime}=\left(\epsilon_{1}, \ldots, \epsilon_{n}\right)}(-1)^{\mu\left(\epsilon^{\prime}\right)}\left(P_{k}^{(\ell)}\left(L_{x, x_{n+1}(+)} ; 1\right)-P_{k}^{(\ell)}\left(L_{x, x_{n+1}(-)} ; 1\right)\right),
\end{aligned}
$$

where $x=\left(x_{1}\left(\epsilon_{1}\right), x_{2}\left(\epsilon_{2}\right), \ldots, x_{n}\left(\epsilon_{n}\right)\right)$. Using (2.5), this becomes

$$
\begin{aligned}
P_{k}^{(\ell)}\left(L_{\times}^{n+1} ; 1\right) & \\
= & \sum_{\epsilon^{\prime}=\left(\epsilon_{1}, \ldots, \epsilon_{n}\right)}(-1)^{\mu\left(\epsilon^{\prime}\right)}\left(2 \ell P_{k}^{(\ell-1)}\left(L_{x, x_{n+1}(-)} ; 1\right)+\ell(\ell-1) P_{k}^{(\ell-2)}\left(L_{x, x_{n+1}(-)} ; 1\right)\right. \\
& \left.+P_{k-1}^{(\ell)}\left(L_{x, x_{n+1}(0)} ; 1\right)+\ell P_{k-1}^{(\ell-1)}\left(L_{x, x_{n+1}(0)} ; 1\right)\right) .
\end{aligned}
$$

Again using (1.2), we have

$$
\begin{aligned}
& P_{k}^{(\ell)}\left(L_{\times}^{n+1} ; 1\right) \\
& \quad=2 \ell P_{k}^{(\ell-1)}\left(L_{-}^{n} ; 1\right)+\ell(\ell-1) P_{k}^{(\ell-2)}\left(L_{-}^{n} ; 1\right)+P_{k-1}^{(\ell)}\left(L_{0}^{n} ; 1\right)+\ell P_{k-1}^{(\ell-1)}\left(L_{0}^{n} ; 1\right) .
\end{aligned}
$$

If $k+\ell=n$, then by the inductive hypothesis, the right-hand side is zero, thereby completing the proof.

If $k+\ell=n+1$, then (2.6) implies the recursion formula:

$$
P_{k}^{(\ell)}\left(\alpha^{n+1}\right)=2 \ell P_{k}^{(\ell-1)}\left(\alpha_{-}^{n}\right)+P_{k-1}^{(\ell)}\left(\alpha_{0}^{n}\right)
$$


where $\alpha^{n+1}, \alpha_{-}^{n}, \alpha_{0}^{n}$ are the configurations respecting $L_{\times}^{n+1}, L_{-}^{n}, L_{0}^{n}$, respectively. Regard $\alpha^{n+1}, \alpha_{-}^{n}, \alpha_{0}^{n}$ as chord-diagrams. Then $\alpha_{-}^{n}$ is obtained from $\alpha^{n+1}$ by deleting the chord $c$ corresponding to the double point $x_{n+1}$, and $\alpha_{0}^{n}$ is obtained from $\alpha^{n+1}$ by chainging the chord $c$ as in Fig. 4. Thus the $P_{k}^{(\ell)}$-value of any configuration of order $k+\ell$ is given as a $\mathbb{Z}$-linear combination of $P_{-r}^{(r)}\left(U^{r+1} ; 1\right)$, which is equal to $r !(-2)^{r}$ by Proposition 6 .
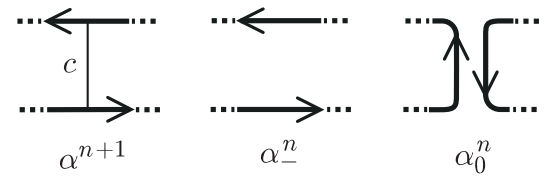

Fig. 4

ExAmPle 1. Let $\sigma^{2}$ and $\tau^{1}$ be the chord-diagrams shown in Fig. 5. Deleting a chord from $\sigma^{2}$, it becomes inadmissible. So using (2.7), we have

$$
P_{0}^{(2)}\left(\sigma^{2}\right)=P_{-1}^{(2)}\left(\tau^{1}\right)=4 P_{-1}^{(1)}\left(U^{2}\right)=-8 .
$$

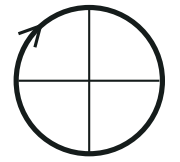

$\sigma^{2}$

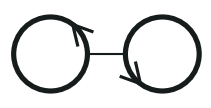

$\tau^{1}$

Fig. 5

The Conway polynomial $\nabla_{L}(z) \in \mathbb{Z}[z][\mathrm{C}]$ of an oriented $r$-component link $L$ is given by

$$
\nabla_{L}(z)=P(L ; 1, z)
$$

and is of the form

$$
\nabla_{L}(z)=\sum_{i=0}^{N} a_{r+2 i-1}(L) z^{r+2 i-1}
$$

where $a_{r+2 i-1}(L) \in \mathbb{Z}$.

From Lemma $1, a_{n}(L)\left(=P_{n}^{(0)}(L ; 1)\right)$ is a Vassiliev link invariant of order $\leq n$. The recursion formula, which follows from $(2.7)$, is easy:

$$
a_{n}\left(\alpha^{n}\right)=a_{n-1}\left(\alpha_{0}^{n-1}\right)
$$

Since

$$
a_{0}\left(U^{r}\right)= \begin{cases}1 & \text { if } r=1 \\ 0 & \text { if } r>1\end{cases}
$$

the $a_{n}$-value of any $n$-configuration is either 1 or 0 .

EXAMPLE 2. Let $\sigma^{n}$ and $\tau^{n-1}$ be the chord-diagrams shown in Fig. 6. Applying (2.8), we have

$$
a_{n}\left(\sigma^{n}\right)=a_{n-1}\left(\tau^{n-1}\right)=a_{n-2}\left(\sigma^{n-2}\right)
$$


Since $a_{1}\left(\sigma^{1}\right)=0$ and $a_{0}\left(\sigma^{0}\right)=a_{0}(U)=1$, we obtain

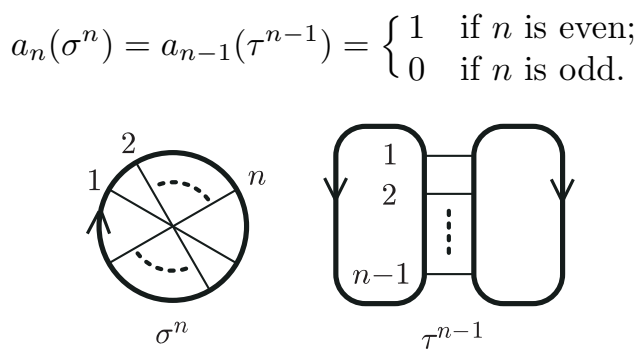

Fig. 6

Let $A_{i}^{n}, n \geq 2,1 \leq i \leq n-1$, be an $n$-configuration for a circle and $B_{i}^{n}, n \geq 1$, $1 \leq i \leq n$, be one for two circles, which are represented by the chord-diagrams shown in Figs. $7(\mathrm{a})$ and $7(\mathrm{~b})$, respectively.

(a)

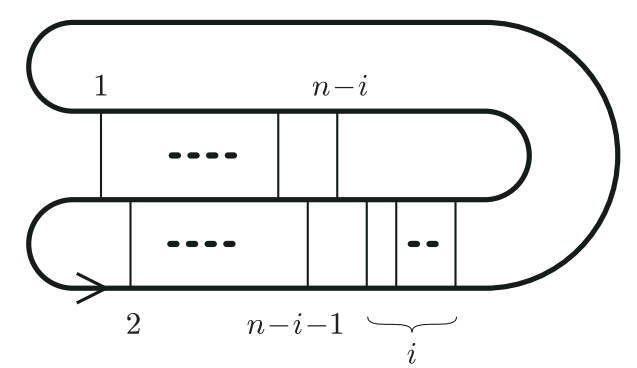

$A_{i}^{n}$ (b)

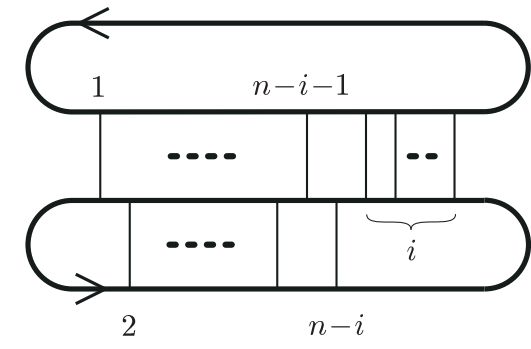

$B_{i}^{n}$

Fig. 7

LEMMA 2. Suppose that $n=k+\ell$.

$$
P_{k}^{(\ell)}\left(A_{i}^{n}\right)= \begin{cases}(i-1) ! 2^{i-1} & \text { if } \ell=i-1 \\ -(i+1) ! 2^{i+1} & \text { if } \ell=i+1 \\ 0 & \text { otherwise }\end{cases}
$$

where $k=0,2, \ldots, 2[n / 2]$.

$$
P_{k}^{(\ell)}\left(B_{i}^{n}\right)= \begin{cases}(i-1) ! 2^{i-1} & \text { if } \ell=i-1 \\ -(i+1) ! 2^{i+1} & \text { if } \ell=i+1 \\ 0 & \text { otherwise }\end{cases}
$$

where $k=-1,1, \ldots, 2[(n+1) / 2]-1$.

Pr o of. First, we consider the case $i=1$. In the same way as Example 1, we have

$$
P_{k}^{(\ell)}\left(A_{1}^{n}\right)=P_{k-1}^{(\ell)}\left(B_{1}^{n-1}\right)=P_{k-2}^{(\ell)}\left(A_{1}^{n-2}\right) .
$$

So if $i=1$, the lemma is true by Examples 1 and 2 .

Suppose that $i>1$. Applying (2.7), we have

$$
P_{k}^{(\ell)}\left(A_{i}^{n}\right)=2 \ell P_{k}^{(\ell-1)}\left(A_{i-1}^{n-1}\right), \quad P_{k}^{(\ell)}\left(B_{i}^{n}\right)=2 \ell P_{k}^{(\ell-1)}\left(B_{i-1}^{n-1}\right),
$$


which are equal to

$$
\begin{aligned}
& 2^{i-1} \frac{\ell !}{(\ell-i+1) !} P_{k}^{(\ell-i+1)}\left(A_{1}^{n-i+1}\right) ; \\
& 2^{i-1} \frac{\ell !}{(\ell-i+1) !} P_{k}^{(\ell-i+1)}\left(B_{1}^{n-i+1}\right),
\end{aligned}
$$

respectively. From the $i=1$ case, we obtain the results.

Let $\gamma^{n-1}$ be an $(n-1)$-configuration, and $c_{1}$ and $c_{2}$ be its two chords. Let $\gamma_{i}^{n}, i=1,2$, be an $n$-configuration obtained from $\gamma^{n-1}$ by adding a new chord parallel to $c_{i}$ as shown in Fig. 8. Applying (2.7) and (2.8), we have immediately

Proposition 7. If $k+\ell=n$, then

$$
P_{k}^{(\ell)}\left(\gamma_{1}^{n}\right)=P_{k}^{(\ell)}\left(\gamma_{2}^{n}\right)
$$

In particular,

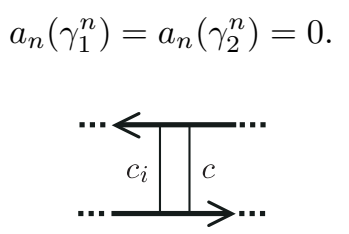

Fig. 8

Let $A^{n}\left(i_{1}, i_{2}, \ldots, i_{p}\right)$ be an $n$-configuration represented by the chord-diagram shown in Fig. 9, where $p$ is even, $i_{1}+i_{2}+\cdots+i_{p}=n$, and $i_{1}, i_{2}, \ldots, i_{p} \geq 1$. When $p=n-j+1$, $i_{1}=i_{2}=\ldots=i_{n-j}=1$ and $i_{n-j+1}=j$, it coincides with $A_{j}^{n}$. Therefore, Proposition 7 implies that if $k+\ell=n$, then

$$
P_{k}^{(\ell)}\left(A^{n}\left(i_{1}, i_{2}, \ldots, i_{n-j+1}\right)\right)=P_{k}^{(\ell)}\left(A_{j}^{n}\right) .
$$

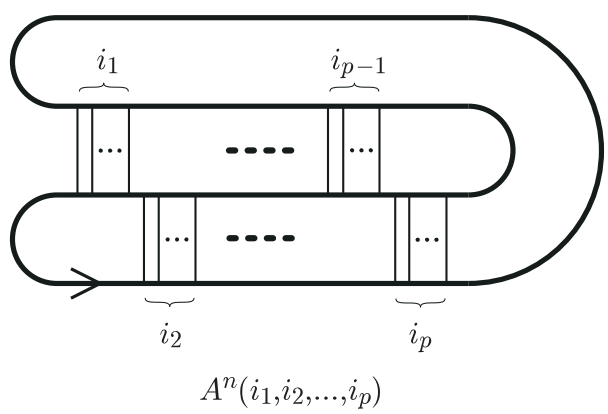

Fig. 9

3. The Jones polynomial. The Jones polynomial $V(L ; t) \in \mathbb{Z}\left[t^{ \pm 1 / 2}\right][\mathrm{J}]$ of an oriented link $L$ is given by

$$
V(L ; t)=P\left(L ; t, t^{1 / 2}-t^{-1 / 2}\right) .
$$


The aim of this section is to prove the following:

ThEOREM 1. $V^{(n)}(L ; 1)$ is a Vassiliev link invariant of order $n$.

Noting that

$$
V(L ; 1)=(-2)^{r-1}
$$

for an $r$-component link $L[\mathrm{~J},(12.1)]$, we can prove the following in the same way as Lemma 1.

LEMma 3. $V^{(n)}(L ; 1)$ is a Vassiliev link invariant of order less than or equal to $n$.

From the proof of Lemma 3, we get the recursion formula which is similar to (2.7) and (2.8):

$$
V^{(n+1)}\left(\alpha^{n+1}\right)=2(n+1) V^{(n)}\left(\alpha_{-}^{n}\right)+(n+1) V^{(n)}\left(\alpha_{0}^{n}\right),
$$

where $\alpha^{n+1}, \alpha_{-}^{n}, \alpha_{0}^{n}$ are the same as in (2.7). Using (3.2) and (3.3), we may calculate the $V^{(n)}$-values of the configurations given in Fig. 7 (cf. Lemma 2):

LEMMA 4.

$$
V^{(n)}\left(A_{i}^{n}\right)=V^{(n)}\left(B_{i}^{n}\right)=-3 \cdot 2^{i-1}(n !) .
$$

Using this, we obtain an analogous result to Proposition 7:

Proposition 8.

$$
V^{(n)}\left(\gamma_{1}^{n}\right)=V^{(n)}\left(\gamma_{2}^{n}\right)=2 n V^{(n-1)}\left(\gamma^{n-1}\right)
$$

This yields an analogous formula to (2.9):

$$
V^{(n)}\left(A^{n}\left(i_{1}, i_{2}, \ldots, i_{n-j+1}\right)\right)=V^{(n)}\left(A_{j}^{n}\right) .
$$

Let $\alpha^{n}$ be an $n$-configuration, and $\alpha^{n} \sqcup U$ denote the $n$-configuration represented by the disjoint union of $\alpha^{n}$ and a circle. Then we have:

LEMMA 5.

$$
V^{(n)}\left(\alpha^{n} \sqcup U\right)=-2 V^{(n)}\left(\alpha^{n}\right) .
$$

Pr oof. If $L$ is a link, then

$$
V(L \sqcup U ; t)=\left(-t^{1 / 2}-t^{-1 / 2}\right) V(L ; t),
$$

and so

$$
V^{(n)}(L \sqcup U ; t)=\sum_{i=0}^{n}\left(-t^{1 / 2}-t^{-1 / 2}\right)^{(i)} V^{(n-i)}(L ; t) .
$$

By Lemma 3, if $k<n$, then

$$
V^{(k)}\left(\alpha^{n}\right)=0
$$

and thus we obtain the result.

Proof of Theorem 1. By Lemma 3, it suffices to show that there exists an $n$-configuration $\alpha^{n}$ for $r$ circles such that $V^{(n)}\left(\alpha^{n}\right) \neq 0$ for each $n$ and $r$. Lemma 4 shows this for $r=1,2$. Note that $V_{1}=V_{0}$. For $r>2$, we have

$$
V^{(n)}\left(B_{i}^{n} \sqcup U^{r-2}\right)=\left(-3 \cdot 2^{i-1}(n !)\right)(-2)^{r-2} \neq 0
$$

by Lemmas 4 and 5 , and thus the proof is complete. 
Remark. Melvin and Morton $[\mathrm{MM}]$ prove Theorem 1 for a knot using the configuration $A_{n-1}^{n}$.

4. The product of Vassiliev link invariants. Let $v$ and $w$ be Vassiliev link invariants. Then the product $v \cdot w$ is defined by $(v \cdot w)(L)=v(L) w(L)$, for a non-singular link $L$

Lemma 6. Let $L^{n}=L_{x_{1}(\times), x_{2}(\times), \ldots, x_{n}(\times)}$ be a singular link with $n$ double points $x_{1}(\times)$, $x_{2}(\times), \ldots, x_{n}(\times)$. Then

$$
(v \cdot w)\left(L^{n}\right)=\sum_{\left(\epsilon_{i}, \epsilon_{i}^{\prime}\right)} v\left(L_{x_{1}\left(\epsilon_{1}\right), x_{2}\left(\epsilon_{2}\right), \ldots, x_{n}\left(\epsilon_{n}\right)}\right) w\left(L_{x_{1}\left(\epsilon_{1}^{\prime}\right), x_{2}\left(\epsilon_{2}^{\prime}\right), \ldots, x_{n}\left(\epsilon_{n}^{\prime}\right)}\right),
$$

where each pair $\left(\epsilon_{i}, \epsilon_{i}^{\prime}\right)$ is either $(+, \times)$ or $(\times,-)$, and the sum runs over the $2^{n}$ possible choices.

Proof. We prove by induction on $n$. When $n=0$, the lemma is just the definition. Suppose that the lemma is true for $n$. By the Vassiliev skein relation (1.1), we have

$$
\begin{aligned}
(v \cdot w) & \left(L_{x_{1}(\times), x_{2}(\times), \ldots, x_{n}(\times), x_{n+1}(\times)}\right) \\
= & (v \cdot w)\left(L_{x_{1}(\times), x_{2}(\times), \ldots, x_{n}(\times), x_{n+1}(+)}\right)-(v \cdot w)\left(L_{x_{1}(\times), x_{2}(\times), \ldots, x_{n}(\times), x_{n+1}(-)}\right) .
\end{aligned}
$$

By the inductive hypothesis, this becomes:

$$
\sum_{\left(\epsilon_{i}, \epsilon_{i}^{\prime}\right)} v\left(L_{x, x_{n+1}(+)}\right) w\left(L_{x^{\prime}, x_{n+1}(+)}\right)-\sum_{\left(\epsilon_{i}, \epsilon_{i}^{\prime}\right)} v\left(L_{x, x_{n+1}(-)}\right) w\left(L_{x^{\prime}, x_{n+1}(-)}\right),
$$

where $x=\left(x_{1}\left(\epsilon_{1}\right), x_{2}\left(\epsilon_{2}\right), \ldots, x_{n}\left(\epsilon_{n}\right)\right)$ and $x^{\prime}=\left(x_{1}\left(\epsilon_{1}^{\prime}\right), x_{2}\left(\epsilon_{2}^{\prime}\right), \ldots, x_{n}\left(\epsilon_{n}^{\prime}\right)\right)$. This is equal to

$$
\begin{aligned}
\sum_{\left(\epsilon_{i}, \epsilon_{i}^{\prime}\right)}\left(v\left(L_{x, x_{n+1}(+)}\right) w\left(L_{x^{\prime}, x_{n+1}(+)}\right)-v\left(L_{x, x_{n+1}(-)}\right) w\left(L_{x^{\prime}, x_{n+1}(-)}\right)\right) \\
=\sum_{\left(\epsilon_{i}, \epsilon_{i}^{\prime}\right)}\left(v\left(L_{x, x_{n+1}(+)}\right) w\left(L_{x^{\prime}, x_{n+1}(+)}\right)-v\left(L_{x, x_{n+1}(+)}\right) w\left(L_{x^{\prime}, x_{n+1}(-)}\right)\right. \\
\\
\left.\quad+v\left(L_{x, x_{n+1}(+)}\right) w\left(L_{x^{\prime}, x_{n+1}(-)}\right)-v\left(L_{x, x_{n+1}(-)}\right) w\left(L_{x^{\prime}, x_{n+1}(-)}\right)\right) \\
=\sum_{\left(\epsilon_{i}, \epsilon_{i}^{\prime}\right)}\left(v\left(L_{x, x_{n+1}(+)}\right)\left(w\left(L_{x^{\prime}, x_{n+1}(+)}\right)-w\left(L_{x^{\prime}, x_{n+1}(-)}\right)\right)\right. \\
\left.\quad+\left(v\left(L_{x, x_{n+1}(+)}\right)-v\left(L_{x, x_{n+1}(-)}\right)\right) w\left(L_{x^{\prime}, x_{n+1}(-)}\right)\right)
\end{aligned}
$$

Again from the Vassiliev skein relation, this becomes

$$
\begin{aligned}
\sum_{\left(\epsilon_{i}, \epsilon_{i}^{\prime}\right)}\left(v\left(L_{x, x_{n+1}(+)}\right) w\left(L_{x^{\prime}, x_{n+1}(\times)}\right)+v(\right. & \left.\left.L_{x, x_{n+1}(\times)}\right) w\left(L_{x^{\prime}, x_{n+1}(-)}\right)\right) \\
& =\sum_{\left(\epsilon_{i}, \epsilon_{i}^{\prime}\right)} v\left(L_{x, x_{n+1}\left(\epsilon_{n+1}\right)}\right) w\left(L_{x^{\prime}, x_{n+1}\left(\epsilon_{n+1}^{\prime}\right)}\right) .
\end{aligned}
$$

We have completed the proof of Lemma 6 . 
This lemma implies immediately the following, which is proved for a knot by Lin (unpublished) and Bar-Natan [BN].

TheOREm 2. If $v$ and $w$ are Vassiliev link invariants of orders less than or equal to $p$ and $q$, respectively, then the product $v \cdot w$ is a Vassiliev link invariant of order less than or equal to $p+q$.

Let $\alpha^{p+q}$ be a chord-diagram of order $p+q$ for $r$ circles, and $C$ the set of its $p+q$ chords. For a subset $S$ of $C$ with $\# S=p$, let $\alpha_{S}^{p}$ denote a chord-diagram of order $p$ consisting of $r$ circles and the chords in $S$.

Proposition 9. Let $v$ and $w$ be Vassiliev link invariants of orders $p$ and $q$, respectively. Then

$$
(v \cdot w)\left(\alpha^{p+q}\right)=\sum_{S \sqcup \bar{S}=C} v\left(\alpha_{S}^{p}\right) w\left(\alpha_{\bar{S}}^{q}\right),
$$

where $S \sqcup \bar{S}$ is the disjoint union of $S$ and $\bar{S}$.

Proof. This follows from Lemma 6 when $n=p+q$.

EXAMPLE 3 . We calculate $a_{2}^{2}\left(=a_{2} \cdot a_{2}\right)$, the square of $a_{2}$, the coefficient of $z^{2}$ in the Conway polynomial. Let $\alpha^{4}$ be a chord-diagram for a circle with $C=\left\{c_{1}, c_{2}, c_{3}, c_{4}\right\}$ a set of its chords. Applying Proposition 9, we have

$$
\begin{aligned}
& a_{2}^{2}\left(\alpha^{4}\right) \\
& =2\left(a_{2}\left(\alpha_{\left\{c_{1}, c_{2}\right\}}^{2}\right) a_{2}\left(\alpha_{\left\{c_{3}, c_{4}\right\}}^{2}\right)+a_{2}\left(\alpha_{\left\{c_{1}, c_{3}\right\}}^{2}\right) a_{2}\left(\alpha_{\left\{c_{2}, c_{4}\right\}}^{2}\right)+a_{2}\left(\alpha_{\left\{c_{1}, c_{4}\right\}}^{2}\right) a_{2}\left(\alpha_{\left\{c_{2}, c_{3}\right\}}^{2}\right)\right) .
\end{aligned}
$$

Using this, we obtain the following:

$$
\begin{aligned}
a_{2}^{2}\left(\sigma^{4}\right) & =6 a_{2}\left(\sigma^{2}\right)^{2}=6 ; \\
a_{2}^{2}\left(A_{1}^{4}\right) & =2 a_{2}\left(\sigma^{2}\right)^{2}=2 ; \\
a_{2}^{2}\left(A_{3}^{4}\right) & =0 \\
a_{2}^{2}\left(A^{4}(2,2)\right) & =4 a_{2}\left(\sigma^{2}\right)^{2}=4,
\end{aligned}
$$

where $\sigma^{2}$ and $\sigma^{4}$ are given in Figs. 5 and 6 (Exapmles 1 and 2), $A_{1}^{4}$ and $A_{3}^{4}$ in Fig. 7, and $A^{4}(2,2)$ in Fig. 9.

R e m a r k. Hoste $[\mathrm{H}]$ gives a formula for $a_{r-1}(L)$ with $L$ an $r$-component link in terms of the linking numbers; more precisely, $a_{r-1}(L)$ is a polynomial of degree $r-1$ in the linking numbers of the sublink of $L$. In particular, if $r=2$, then $a_{1}(L)$ is the linking number of $L$ (cf. [Kf, p. 24]). By Theorem 2 and the result in [S2], we see that $a_{r-1}(L)$ is a Vassiliev invariant of order less than or equal to $r-1$.

5. Vassiliev knot invariants of order $\leq 4$. In this section, we study a Vassiliev knot invariant of order $\leq 4$, making use of the result of Birman and Lin [BL, Example 3.9].

The only admissible 2-configuration is $\sigma^{2}$ shown in Fig. 5, which are denoted by the symbol 22 in [BL]. Let $M^{2}$ be the singular knot of order 2 shown in Fig. 10 respecting it.

There are two admissible 3-configurations: $A_{2}^{3}$ (Fig. 7) and $\sigma^{3}$ (Fig. 6). In [BL], they are denoted by the symbols $\mathbf{2 3 2}$ and $\mathbf{3 3 3}$, respectively, and it is shown that if $v_{3}$ is a 


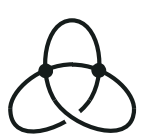

$M^{2}$

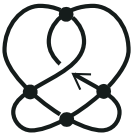

$M_{1}^{4}$

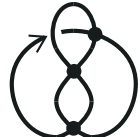

$M_{1}^{3}$

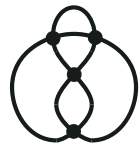

$M_{2}^{4}$

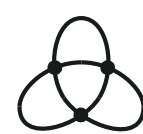

$M_{2}^{3}$

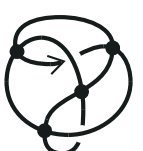

$M_{3}^{4}$

Fig. 10

Vassiliev invariant of order 3, then

$$
v_{3}\left(\sigma^{3}\right)=2 v_{3}\left(A_{2}^{3}\right) .
$$

Let $M_{1}^{3}$ and $M_{2}^{3}$ be the singular knots of order 3 shown in Fig. 10 respecting $A_{2}^{3}$ and $\sigma^{3}$.

Let $v$ be a Vassiliev knot invariant of order $\leq 4$ and $K$ be a knot. There are seven admissible 4-configurations, and the $v$-value of any 4-configuration is determined by those of the three 4-configurations $A_{3}^{4}, A^{4}(2,2), A_{1}^{4}$ shown in Figs. 7 and 9. They are denoted by the symbols $\mathbf{2 4 4 2}, \mathbf{3 5 3 3}$, and $\mathbf{2 3 3 2}$, respectively in [BL]. Let $M_{1}^{4}, M_{2}^{4}, M_{3}^{4}$ be the singular knots of order 4 shown in Fig. 10 respecting them.

Therefore, Proposition 4 implies

$$
v(K)=\left[\begin{array}{llllll}
v(U) & v\left(M^{2}\right) & v\left(M_{1}^{3}\right) & v\left(M_{1}^{4}\right) & v\left(M_{2}^{4}\right) & v\left(M_{3}^{4}\right)
\end{array}\right]\left[\begin{array}{c}
1 \\
p \\
q \\
r_{1} \\
r_{2} \\
r_{3}
\end{array}\right],
$$

where $p, q, r_{1}, r_{2}, r_{3}$ are integers.

Let $3_{1}, 4_{1}, 5_{1}, 5_{2}$ be the knots in the table of [R]. We denote by $K$ ! the mirror image of the knot $K$. So $3_{1}$ and $3_{1}$ ! denote the left- and right-hand trefoil knots, respectively, and $4_{1}$ is the figure-eight knot. Using the Vassiliev skein relatin (1.1), we have

$$
\begin{aligned}
& {\left[\begin{array}{llllll}
v\left(M^{2}\right) & v\left(M_{1}^{3}\right) & v\left(M_{1}^{4}\right) & v\left(M_{2}^{4}\right) & v\left(M_{3}^{4}\right)
\end{array}\right]} \\
& \quad=\left[\begin{array}{llllll}
v(U) & v\left(3_{1} !\right) & v\left(3_{1}\right) & v\left(4_{1}\right) & v\left(5_{1} !\right) & v\left(5_{2} !\right)
\end{array}\right]\left[\begin{array}{ccccc}
-1 & -2 & 3 & -4 & 0 \\
1 & 1 & -3 & 1 & 1 \\
0 & 0 & 0 & 1 & 0 \\
0 & 1 & -1 & 2 & 0 \\
0 & 0 & 0 & 0 & 1 \\
0 & 0 & 1 & 0 & -2
\end{array}\right]
\end{aligned}
$$

In Table 1, we give the values of the Vassiliev invariants of order less than or equal to 4 derived from the HOMFLY and the Jones polynomials. Many of them are already given in Examples 1-3. 


\begin{tabular}{|c|rrrrrrrrrrrr|}
\hline & $a_{2}$ & $P_{0}^{(2)}$ & $V^{(2)}$ & $P_{2}^{(1)}$ & $\frac{P_{0}^{(3)}}{24}$ & $\frac{V^{(3)}}{18}$ & $a_{4}$ & $P_{2}^{(2)}$ & $\frac{P_{0}^{(4)}}{24}$ & $\frac{V^{(4)}}{24}$ & $a_{2}^{2}$ \\
\hline $3_{1} !$ & 1 & -8 & -6 & 2 & -1 & -1 & 0 & 2 & -1 & -1 & 1 \\
$4_{1}$ & -1 & 8 & 6 & 0 & -1 & -1 & 0 & 0 & 5 & 4 & 1 \\
\hline$M^{2}$ & 1 & -8 & -6 & 2 & -1 & -1 & 0 & 2 & -1 & -1 & 1 \\
$M_{1}^{3}$ & 0 & 0 & 0 & 2 & -2 & -2 & 0 & 2 & 4 & 3 & 2 \\
$M_{1}^{4}$ & 0 & 0 & 0 & 0 & 0 & 0 & 0 & 8 & -16 & -12 & 0 \\
$M_{2}^{4}$ & 0 & 0 & 0 & 0 & 0 & 0 & 0 & 8 & -16 & -12 & 4 \\
$M_{3}^{4}$ & 0 & 0 & 0 & 0 & 0 & 0 & 1 & -8 & 0 & -3 & 2 \\
\hline
\end{tabular}

Table 1

From (5.2) and Table 1, we have:

$$
\left[\begin{array}{c}
a_{2}(K) \\
P_{0}^{(3)}(K ; 1) / 24 \\
a_{2}(K)^{2} \\
a_{4}(K) \\
P_{0}^{(4)}(K ; 1) / 24
\end{array}\right]=\left[\begin{array}{ccccc}
1 & 0 & 0 & 0 & 0 \\
-1 & 2 & 0 & 0 & 0 \\
1 & 2 & 0 & 4 & 2 \\
0 & 0 & 0 & 0 & 1 \\
-1 & 4 & -16 & -16 & 0
\end{array}\right]\left[\begin{array}{l}
p \\
q \\
r_{1} \\
r_{2} \\
r_{3}
\end{array}\right]
$$

and thus we have

$$
\left[\begin{array}{l}
p \\
q \\
r_{1} \\
r_{2} \\
r_{3}
\end{array}\right]=\left[\begin{array}{ccccc}
1 & 0 & 0 & 0 & 0 \\
-1 / 2 & -1 / 2 & 0 & 0 & 0 \\
-3 / 16 & -3 / 8 & -1 / 4 & 1 / 2 & -1 / 16 \\
0 & 1 / 4 & 1 / 4 & -1 / 2 & 0 \\
0 & 0 & 0 & 1 & 0
\end{array}\right]\left[\begin{array}{c}
a_{2}(K) \\
P_{0}^{(3)}(K ; 1) / 24 \\
a_{2}(K)^{2} \\
a_{4}(K) \\
P_{0}^{(4)}(K ; 1) / 24
\end{array}\right]
$$

Also we have:

$$
\left[\begin{array}{c}
P_{0}^{(2)}(K ; 1) \\
V_{K}^{(2)}(1) \\
P_{2}^{(1)}(K ; 1) \\
V_{K}^{(3)}(1) / 18 \\
P_{2}^{(2)}(K ; 1) \\
V_{K}^{(4)}(1) / 24
\end{array}\right]=\left[\begin{array}{ccccc}
-8 & 0 & 0 & 0 & 0 \\
-6 & 0 & 0 & 0 & 0 \\
2 & 2 & 0 & 0 & 0 \\
-1 & -2 & 0 & 0 & 0 \\
2 & 2 & 8 & 8 & -8 \\
-1 & 3 & -12 & -12 & -3
\end{array}\right]\left[\begin{array}{l}
p \\
q \\
r_{1} \\
r_{2} \\
r_{3}
\end{array}\right]
$$

Substituting (5.4) to (5.5), we obtain:

$$
\begin{aligned}
P_{0}^{(2)}(K ; 1) & =-8 a_{2}(K) ; \\
V_{K}^{(2)}(1) & =-6 a_{2}(K) \quad([\mathrm{Mu} 1]) ; \\
P_{2}^{(1)}(K ; 1) & =a_{2}(K)-\frac{1}{24} P_{0}^{(3)}(K ; 1) \quad([\mathrm{Mi}]) ; \\
V_{K}^{(3)}(1) & =\frac{3}{4} P_{0}^{(3)}(K ; 1) \quad([\mathrm{Mi}]) ; \\
P_{2}^{(2)}(K ; 1) & =-\frac{1}{2} a_{2}(K)-\frac{1}{12} P_{0}^{(3)}(K ; 1)-8 a_{4}(K)-\frac{1}{48} P_{0}^{(4)}(K ; 1) ; \\
V_{K}^{(4)}(1) & =-6 a_{2}(K)-72 a_{4}(K)+18 P_{0}^{(4)}(K ; 1) .
\end{aligned}
$$


Combining (5.2)-(5.4), we obtain:

$$
v(K)=\left[\begin{array}{llllll}
v(U) & v\left(3_{1} !\right) & v\left(3_{1}\right) & v\left(4_{1}\right) & v\left(5_{1} !\right) & v\left(5_{2} !\right)
\end{array}\right] X\left[\begin{array}{c}
1 \\
a_{2}(K) \\
P_{0}^{(3)}(K ; 1) / 24 \\
a_{2}(K)^{2} \\
a_{4}(K) \\
P_{0}^{(4)}(K ; 1) / 24
\end{array}\right],
$$

where

$$
X=\left[\begin{array}{cccccc}
1 & -9 / 16 & -9 / 8 & -7 / 4 & 7 / 2 & -3 / 16 \\
0 & 17 / 16 & 7 / 8 & 1 & -1 & 3 / 16 \\
0 & 0 & 1 / 4 & 1 / 4 & -1 / 2 & 0 \\
0 & -5 / 16 & 3 / 8 & 3 / 4 & -3 / 2 & 1 / 16 \\
0 & 0 & 0 & 0 & 1 & 0 \\
0 & -3 / 16 & -3 / 8 & -1 / 4 & -3 / 2 & -1 / 16
\end{array}\right] .
$$

We consider $v_{2}$, a Vassiliev knot invariant of order $\leq 2$. Then (5.2) becomes

$$
v_{2}(K)=v_{2}(U)+p v_{2}\left(M^{2}\right) .
$$

Then using (5.3) and (5.4), we have

$$
v_{2}(K)=\left[v_{2}(U) \quad v_{2}\left(3_{1} !\right)\right]\left[\begin{array}{cc}
1 & -1 \\
0 & 1
\end{array}\right]\left[\begin{array}{c}
1 \\
a_{2}(K)
\end{array}\right] .
$$

This is given in [La, Proposition 4.2.9], where $V_{2}(K)=a_{2}(K)$ and $v_{2}(U)$ is determined to be zero.

Next, we consider $v_{3}$, a Vassiliev knot invariant of order $\leq 3$. Then (5.2) becomes

$$
v_{3}(K)=v_{3}(U)+p v_{3}\left(M^{2}\right)+q v_{3}\left(M_{1}^{3}\right) .
$$

Then using (5.3) and (5.4), we have

$$
v_{3}(K)=\left[\begin{array}{lll}
v_{3}(U) & v_{3}\left(3_{1} !\right) & v_{3}\left(4_{1}\right)
\end{array}\right]\left[\begin{array}{ccc}
1 & 0 & 1 \\
0 & 1 / 2 & -1 / 2 \\
0 & -1 / 2 & -1 / 2
\end{array}\right]\left[\begin{array}{c}
1 \\
a_{2}(K) \\
P_{0}^{(3)}(K ; 1) / 24
\end{array}\right]
$$

Substituting (5.8) to (5.16), we obtain

$$
v_{3}(K)=\left[\begin{array}{lll}
v_{3}(U) & v_{3}\left(3_{1} !\right) & v_{3}\left(4_{1}\right)
\end{array}\right]\left[\begin{array}{ccc}
1 & 1 & -1 \\
0 & 0 & 1 / 2 \\
0 & -1 & 1 / 2
\end{array}\right]\left[\begin{array}{c}
1 \\
a_{2}(K) \\
P_{2}^{(1)}(K ; 1)
\end{array}\right],
$$

which is the first formula in [La, Proposition 4.3.10] with $V_{3}(K)=P_{2}^{(1)}(K ; 1) / 2$.

Substituting (5.1) to (5.2), we have

$$
v_{3}(K)=v_{3}(U)+p v_{3}\left(M^{2}\right)+\frac{q}{2} v_{3}\left(M_{2}^{3}\right) .
$$

Using the Vassiliev skein relation (1.1), we have

$$
v_{3}\left(M_{2}^{3}\right)=v_{3}\left(3_{1} !\right)-v_{3}\left(3_{1}\right),
$$

and thus we obtain

$$
v_{3}(K)=\left[\begin{array}{lll}
v_{3}(U) & v_{3}\left(3_{1} !\right) & v_{3}\left(3_{1}\right)
\end{array}\right]\left[\begin{array}{ccc}
1 & -1 & 0 \\
0 & 3 / 4 & -1 / 4 \\
0 & 1 / 4 & 1 / 4
\end{array}\right]\left[\begin{array}{c}
1 \\
a_{2}(K) \\
P_{0}^{(3)}(K ; 1) / 24
\end{array}\right] .
$$


Substituting (5.8) to (5.20), we obtain the second formula in [La, Proposition 4.3.10]:

$$
v_{3}(K)=\left[\begin{array}{lll}
v_{3}(U) & v_{3}\left(3_{1} !\right) & v_{3}\left(3_{1}\right)
\end{array}\right]\left[\begin{array}{ccc}
1 & -1 & 0 \\
0 & 1 / 2 & 1 / 4 \\
0 & 1 / 2 & -1 / 4
\end{array}\right]\left[\begin{array}{c}
1 \\
a_{2}(K) \\
P_{2}^{(1)}(K ; 1)
\end{array}\right] .
$$

6. A relation among $P_{k}^{(\ell)}$. From (5.6), (5.8) and (5.10), we have

$$
\begin{gathered}
\frac{1}{2 ! 2^{2}} P_{0}^{(2)}(K ; 1)+a_{2}(K)=0 ; \\
\frac{1}{3 ! 2^{3}} P_{0}^{(3)}(K ; 1)+\frac{1}{2} P_{2}^{(1)}(K ; 1)=\frac{1}{2} a_{2}(K) ; \\
\frac{1}{4 ! 2^{4}} P_{0}^{(4)}(K ; 1)+\frac{1}{2 ! 2^{2}} P_{2}^{(2)}(K ; 1)+a_{4}(K)=-\frac{5}{16} a_{2}(K)+\frac{1}{4} P_{2}^{(1)}(K ; 1) .
\end{gathered}
$$

We can generalize these formulas. Let $\varphi_{m}$ be a Vassiliev invariant for an $r$-component link $L$ defined by

$$
\varphi_{k-r+1}(L)=\sum_{i=0}^{[k / 2]} \frac{1}{(k-2 i) ! 2^{k-2 i}} P_{2 i-r+1}^{(k-2 i)}(L ; 1) .
$$

By Lemma 1, $\varphi_{k-r+1}$ is a Vassiliev link invariant of order $\leq \max \{k-r+1,0\}$. However, we shall prove:

THEOREM 3. $\varphi_{k-r+1}, k=0,1,2, \ldots$, is a Vassiliev invariant for an $r$-component link of order less than or equal to $\max \{k-r, 0\}$.

In order to prove Theorem 3, we study a Vassiliev link invariant of order less than or equal to one, which may be derived from [Mu2]. The only admissible 1-configuration for an $r$ circles, $r \geq 2$, is represented by the union of $\tau^{1}$ (Fig. 4) and $r-2$ circles, $\tau^{1} \sqcup U^{r-2}$. Using this, we show the following:

Proposition 10. Let $v$ be a Vassiliev invariant of order less than or equal to one for an $r$-component link, $r \geq 2$. Then for an $r$-component link $L$, it holds that

$$
v(L)=v\left(U^{r}\right)+\lambda v\left(\tau^{1} \sqcup U^{r-2}\right),
$$

where $\lambda$ is the total linking number of $L$.

Pr o of. From Proposition 4, we have

$$
v(L)=v\left(U^{r}\right)+m v\left(\tau^{1} \sqcup U^{r-2}\right),
$$

where $m$ is an integer. Since $V^{(1)}(L ; 1)$ is a Vassiliev invariant of order $\leq 1$, we have

$$
V^{(1)}(L ; 1)=V^{(1)}\left(U^{r} ; 1\right)+m V^{(1)}\left(\tau^{1} \sqcup U^{r-2}\right) .
$$

By applying (3.3), this becomes

$$
V^{(1)}(L ; 1)=V^{(1)}\left(U^{r} ; 1\right)+2 m V\left(U^{r} ; 1\right)+m V\left(U^{r-1} ; 1\right) .
$$

Using (3.2) and $V^{(1)}(L ; 1)=-3(-2)^{r-2} \lambda[\mathrm{J},(12.2)$; Mu1], we obtain

$$
-3(-2)^{r-2} \lambda=2 m(-2)^{r-1}+m(-2)^{r-2},
$$

from which we get $m=\lambda$, and the proof is complete. 
Proof of Theorem 3. We prove by induction on $k$. If $k \leq r-1$, then this follows from Lemma 1. We show that $\varphi_{1}$ is of order zero. Let $L$ be an $r$-component link. If $r=1$, then

$$
\varphi_{1}(L)=\frac{1}{2} P_{0}^{(1)}(L ; 1)=0 .
$$

Suppose that $r \geq 2$. Since the order of $\varphi_{1}$ is $\leq 1$, from Proposition 10, we have

$$
\varphi_{1}(L)=\varphi_{1}\left(U^{r}\right)+\lambda \varphi_{1}\left(\tau^{1} \sqcup U^{r-2}\right) .
$$

Using (2.7), we have

$$
P_{2 i-r+1}^{(r-2 i)}\left(\tau^{1} \sqcup U^{r-2}\right)=2(r-2 i) P_{2 i-r+1}^{(r-2 i-1)}\left(U^{r} ; 1\right)+P_{2 i-r}^{(r-2 i)}\left(U^{r-1} ; 1\right),
$$

and so

$$
\varphi_{1}\left(\tau^{1} \sqcup U^{r-2}\right)=\sum_{i=0}^{[r / 2]}\left(\frac{P_{2 i-r+1}^{(r-2 i-1)}\left(U^{r} ; 1\right)}{(r-2 i-1) ! 2^{r-2 i-1}}+\frac{P_{2 i-r}^{(r-2 i)}\left(U^{r-1} ; 1\right)}{(r-2 i) ! 2^{r-2 i}}\right),
$$

which is zero by Proposition 6 . Therefore, $\varphi_{1}$ is a constant map.

From (2.2), we have

$$
P(L ; y+1, y)=\sum_{i=0}^{n} P_{2 i-r+1}(L ; y+1) y^{2 i-r+1} .
$$

We expand $P_{2 i-r+1}(L ; y+1)$ via its Taylor series:

$$
P_{2 i-r+1}(L ; y+1)=\sum_{j=0}^{\infty} \frac{P_{2 i-r+1}^{(j)}(L ; 1)}{j !} y^{j} .
$$

Then we obtain a power series expansion of $P(L ; y+1, y)$ :

$$
P(L ; y+1, y)=\sum_{k=0}^{\infty} \Phi_{k-r+1}(L) y^{k-r+1},
$$

where

$$
\Phi_{k-r+1}(L)=2^{k-r+1} \varphi_{k-r+1}(L) .
$$

The equation (2.1b) implies

$$
\begin{aligned}
P\left(L_{+} ; y+1,2 y\right)-P\left(L_{-} ; y+1,2 y\right) & \\
\quad & \left(y^{2}+2 y\right) P\left(L_{-} ; y+1,2 y\right)+2\left(y^{2}+y\right) P\left(L_{0} ; y+1,2 y\right) .
\end{aligned}
$$

Then from (6.3), we have

$$
\Phi_{\ell}\left(L_{+}\right)-\Phi_{\ell}\left(L_{-}\right)=\Phi_{\ell-2}\left(L_{-}\right)+2 \Phi_{\ell-1}\left(L_{-}\right)+2 \Phi_{\ell-2}\left(L_{0}\right)+2 \Phi_{\ell-1}\left(L_{0}\right) .
$$

Assume that $\Phi_{k}$ is a Vassiliev invariant of order $\leq \max \{k-1,0\}$ for each $k(<\ell)$. Then using (6.6), we can prove that $\Phi_{\ell}(L)$ is of order $\leq \ell-1$ in a similar way to the proof of Lemma 1. This completes the proof of Theorem 3.

From this theorem, for a knot $K$ we have

$$
\sum_{i=0}^{[n / 2]} \frac{1}{(n-2 i) ! 2^{n-2 i}} P_{2 i}^{(n-2 i)}(K ; 1) \equiv 0
$$

in $V_{n} / V_{n-1}$. Therefore, by Lemma 2 (i), we obtain: 
THEOREM 4. The dimension of the subspace of $V_{n} / V_{n-1}$ spanned by the following Vassiliev invariants of order $n$ is $[n / 2]$ :

$$
P_{2 i}^{(n-2 i)}(K ; 1), \quad i=0,1, \ldots,[n / 2] .
$$

Now we reconsider the Jones polynomial of a knot $K$. From (2.2) and (3.1), we have

$$
V(K ; t)=\sum_{k=0}^{N} \psi_{k}(t) P_{2 k}(K ; t),
$$

where $\psi_{k}(t)=\left(t^{1 / 2}-t^{-1 / 2}\right)^{2 k}$. Then we obtain

Since

$$
V^{(n)}(K ; t)=\sum_{k=0}^{N}\left(\sum_{i=0}^{n}\left(\begin{array}{c}
n \\
i
\end{array}\right) \psi_{k}^{(i)}(t) P_{2 k}^{(n-i)}(K ; t)\right) .
$$

we obtain

$$
\psi_{k}^{(i)}(1)= \begin{cases}0 & \text { if } i<2 k \\ (-1)^{i} \frac{i !(i-k-1) !}{(i-2 k) !(k-1) !} & \text { if } i \geq 2 k\end{cases}
$$

$$
\begin{aligned}
V^{(n)}(K ; 1) & =P_{0}^{(n)}(K ; 1)+\sum_{k=1}^{[n / 2]}\left(\sum_{i=2 k}^{n}\left(\begin{array}{c}
n \\
i
\end{array}\right) \psi_{k}^{(i)}(1) P_{2 k}^{(n-i)}(K ; 1)\right) \\
& =P_{0}^{(n)}(K ; 1)+\sum_{k=1}^{[n / 2]}\left(\sum_{i=2 k}^{n}(-1)^{i} \frac{n !(i-k-1) !}{(n-i) !(i-2 k) !(k-1) !} P_{2 k}^{(n-i)}(K ; 1)\right)
\end{aligned}
$$

In particular, we obtain

$$
\begin{aligned}
& V_{K}^{(2)}(1)=P_{0}^{(2)}(K ; 1)+2 a_{2}(K) \\
& V_{K}^{(3)}(1)=P_{0}^{(3)}(K ; 1)+6 P_{2}^{(1)}(K ; 1)-6 a_{2}(K) ; \\
& V_{K}^{(4)}(1)=P_{0}^{(4)}(K ; 1)+12 P_{2}^{(2)}(K ; 1)-24 P_{2}^{(1)}(K ; 1)+24 a_{2}(K)+24 a_{4}(K),
\end{aligned}
$$

cf. (5.6)-(5.11). Furthermore, combining with Theorem 3, we have

$$
V^{(n)}(K ; 1) \equiv \sum_{k=1}^{[n / 2]} \frac{n !\left(1-2^{2 k}\right)}{(n-2 k) !} P_{2 k}^{(n-2 k)}(K ; 1)
$$

in $V_{n} / V_{n-1}$.

7. The link case. Let $V_{n}^{r}$ denote the vector space consisting of all Vassiliev invariants for an $r$-component link of order less than or equal to $n$. We consider the subspace of $V_{n}^{r}$ that is spanned by

$$
P_{2 i-r+1}^{(n+r-2 i-1)}, \quad i=0,1, \ldots,\left[\frac{n+r-1}{2}\right] .
$$

By Theorem 3, these are linearly dependent in $V_{n}^{r} / V_{n-1}^{r}$. If $r-n \geq 3$, then by Proposition $6, P_{2 i-r+1}^{(n+r-2 i-1)}$ is a zero-map for $i=n+1, \ldots,[(n+r-1) / 2]$.

THEOREM 5. Let $s=s(n, r)=\min \{n,[(n+r-1) / 2]\}$ and $r \geq 2$. The dimension of the subspace of $V_{n}^{r} / V_{n-1}^{r}$ spanned by the following Vassiliev invariants of order $n$ is $s$ :

$$
P_{2 i-r+1}^{(n+r-2 i-1)}, \quad i=0,1, \ldots, s .
$$


We prove this theorem by making use of the space of singular links, which is dual to the space of Vassiliev link invariants. This space, which we denote by $\left(V_{n}^{r}\right)^{*}$, is the vector space over $\mathbb{Q}$ generated by equivalent classes of $r$-component singular links, subject to the following relations:

$$
L_{\times}=L_{+}-L_{-}
$$

$$
L=0 \text { if } L \text { has more than } n \text { vertices. }
$$

First we consider the knot case. Put

$$
e_{j}=\frac{1}{(n-2 j) ! 2^{n-2 j}}\left(A_{n-1}^{n}+A_{n-3}^{n}+\cdots+A_{n-2 j+1}^{n}\right) .
$$

Then from Lemma 2 (i), we have

$$
P_{2 i}^{(n-2 i)}\left(e_{j}\right)=\delta_{i j},
$$

where $i, j=1,2, \ldots,[n / 2]$ and $\delta_{i j}$ denotes the Kronecker delta. Namely, $e_{1}, e_{2}, \ldots, e_{s}$ is the dual basis of $P_{2}^{(n-2)}, P_{4}^{(n-4)}, \ldots, P_{2 s}^{(n-2 s)}$, where $s=[n / 2]$. Note that $P_{0}^{(1)}$ is a zero-map.

The following is analogous to Lemma 5.

Lemma 7. Suppose that $k+\ell=n$. Then

$$
P_{k}^{(\ell)}\left(\alpha^{n} \sqcup U\right)=-2 \ell P_{k+1}^{(\ell-1)}\left(\alpha^{n}\right) .
$$

We denote by $\alpha^{n} \vdash U$ an $(n+1)$-configuration obtained by joining $\alpha^{n}$ and a circle $U$ with a new chord.

Lemma 8. Suppose that $k+\ell=n+1$. Then

$$
P_{k}^{(\ell)}\left(\alpha^{n} \vdash U\right)=-4 \ell(\ell-1) P_{k+1}^{(\ell-2)}\left(\alpha^{n}\right)+P_{k-1}^{(\ell)}\left(\alpha^{n}\right) .
$$

Pro of. Applying (2.7), we have

$$
P_{k}^{(\ell)}\left(\alpha^{n} \vdash U\right)=2 \ell P_{k}^{(\ell-1)}\left(\alpha^{n} \sqcup U\right)+P_{k-1}^{(\ell)}\left(\alpha^{n}\right) .
$$

Using Lemma 7, we obtain the result.

Proof of Theorem 5. It is sufficient to prove: There exist vectors $e_{j}$ in $\left(V_{n}^{r}\right)^{*}$ such that

$$
P_{2 i-r+1}^{(n+r-2 i-1)}\left(e_{j}\right)=\delta_{i j}, \quad P_{1-r}^{(n+r-1)}\left(e_{1}\right) \neq 0
$$

where $i, j=1,2, \ldots, s$.

We shall use induction on $r(\geq 2)$. Put

$$
e_{j}=\frac{1}{(n-2 j+1) ! 2^{n-2 j+1}}\left(B_{n}^{n}+B_{n-2}^{n}+\cdots+B_{n-2 j+2}^{n}\right) .
$$

Then from Lemma 2 (ii), we have

$$
P_{2 i-1}^{(n-2 i+1)}\left(e_{j}\right)=\delta_{i j},
$$

where $i, j=1,2, \ldots,[(n+1) / 2]$. Also

$$
P_{-1}^{(n+1)}\left(e_{1}\right)=\frac{-(n+1) ! 2^{n+1}}{(n-1) ! 2^{n-1}}=-4 n(n+1) .
$$

Thus the statement holds for $r=2$. 
Suppose that the statement holds for an $r$-component singular link. Put $s=s(n, r)$. We have two cases:

(i) $s(n, r+1)=s$.

(ii) $s(n, r+1)=s+1$.

CASE (i). First note that $n+r$ is odd. Using Lemma 7, we have

$$
\begin{aligned}
P_{2 i-(r+1)+1}^{(n+(r+1)-2 i-1)}\left(e_{j} \sqcup U\right) & =P_{2 i-r}^{(n+r-2 i)}\left(e_{j} \sqcup U\right) \\
& =-2(n+r-2 i) P_{2 i-r+1}^{(n+r-2 i-1)}\left(e_{j}\right) \\
& =-2(n+r-2 i) \delta_{i j} ; \\
P_{1-(r+1)}^{(n+(r+1)-1)}\left(e_{1} \sqcup U\right) & =P_{-r}^{(n+r)}\left(e_{1} \sqcup U\right) \\
& =-2(n+r) P_{1-r}^{(n+r-1)}\left(e_{1}\right) \\
& \neq 0,
\end{aligned}
$$

where $i, j=1,2, \ldots, s$. Thus

$$
\frac{-1}{2(n+r-2 j)}\left(e_{j} \sqcup U\right), \quad j=1,2, \ldots, s
$$

is the desired vectors in $\left(V_{n}^{r+1}\right)^{*}$.

CASE (ii). The codition yields that $n+r$ is even and $n \geq(n+r) / 2=s+1$. We have $P_{2 i-(r+1)+1}^{(n+(r+1)-2 i-1)}\left(e_{j} \sqcup U\right)$

$$
= \begin{cases}-2(n+r-2 i) \delta_{i j} & \text { if } i, j=1,2, \ldots, s \\ -2(n+r) P_{1-r}^{(n+r-1)}\left(e_{1}\right)(\neq 0) & \text { if } i=0, j=1 ; \\ P_{n}^{(0)}\left(e_{j} \sqcup U\right)=0 & \text { if } i=s+1, j=1,2, \ldots, s .\end{cases}
$$

Since $s(n-1, r)=s$, by the inductive hypothesis, there exists $f \in\left(V_{n-1}^{r}\right)^{*}$ such that $P_{n-1}^{(0)}(f)=1$. Then using Lemma 8 , we have $P_{n}^{(0)}(f \vdash U)=P_{n-1}^{(0)}(f)=1$. Thus from $e_{j} \sqcup U(j=1,2, \ldots, s)$ and $f \vdash U$, we can construct desired set of vectors in $\left(V_{n}^{r+1}\right)^{*}$.

\section{References}

[BN] D. Bar-Natan, On the Vassiliev knot invariants, Topology 34 (1995), 423-472.

[B1] J. S. Birman, New points of view in knot theory, Bull. Amer. Math. Soc. 28 (1993), 253-287.

[B2] J. S. Birman, On the combinatorics of Vassiliev invariants, Braid group, knot theory and statistical mechanics II (M. L. Ge and C. N. Yang, eds.), Advanced Series in Mathematical Physics, World Scientific, Singapore-New Jersey-LondonHong Kong 1994, pp. 1-19.

[BL] J. S. Birman and X.-S. Lin, Knot polynomials and Vassiliev's invariants, Invent. Math. 111 (1993), 225-270.

[CD] S. V. Chmutov and S. V. Duzhin, An upper bound for the number of Vassiliev knot invariants, J. Knot Theory Ramifications 3 (1994), 141-151.

[C] J. H. Conway, An enumeration of knots and links, Computational Problems in Abstract Algebra (J. Leech, ed.), Pergamon Press, New York, 1969, pp. 329-358. 
[FYHLMO] P. Freyd, D. Yetter, J. Hoste, W. B. R. Lickorish, K. Millett and A. Ocneanu, A new polynomial invariant of knots and links, Bull. Amer. Math. Soc. 12 (1985), 239-246.

[G] M. N. Gusarov, A new form of the Conway-Jones polynomial of oriented links, Zap. Nauchn. Sem. Len. Otdel Mat. Inst. Steklov (LOMI) 193 (1991), Geom. i Topol. 1, 4-9; English translation: Topology of manifolds and varieties, Advances in Soviet Mathematics. Vol. 18, Amer. Math. Soc., Providence, RI, 1994, pp. $167-172$

[H] J. Hoste, The first coefficient of the Conway polynomial, Proc. Amer. Math. Soc. 95 (1985), 299-302.

[J] V. F. R. Jones, Hecke algebra representations of braid groups and link polynomials, Ann. Math. 126 (1987), 335-388.

[Kf] L. H. Kauffman, On Knots, Ann. of Math. Studies 115, Princeton Univ. Press, Princeton, 1987.

[Kw] A. Kawauchi, On coefficient polynomials of the skein polynomial of an oriented link, Kobe J. Math. 11 (1994), 49-68.

[L] J. Lannes, Sur les invariants de Vassiliev de degré inférieur ou égal à 3, Enseign. Math. (2) 39 (1993), 295-316.

[LM] W. B. R. Lickorish, and K. C. Millett, A polynomial invariant of oriented links, Topology 26 (1987), 107-141.

[MM] P. Melvin and H. R. Morton, The coloured Jones function, Commun. Math. Phys. 169 (1995), 501-520.

[Me] G. Meng, Bracket models for weight systems and the universal Vassiliev invariants, Topology Appl. 76 (1997), 47-60.

[Mi] Y. Miyazawa, The third derivative of the Jones polynomial, J. Knot Theory Ramifications, (to appear).

[Mu1] H. Murakami, On derivatives of the Jones polynomial, Kobe J. Math. 3 (1986), 61-64.

[Mu2] H. Murakami, Vassiliev type invariant of order two for a link, Proc. Amer. Math. Soc. 124 (1996), 3889-3896.

[N] K. Y. Ng, Groups of ribbon knots, preprint.

[PT] J. H. Przytycki and P. Traczyk, Invariants of links of Conway type, Kobe J. Math. 4 (1987), 115-139.

[R] D. Rolfsen, Knots and Links, Lecture Series no. 7, Publish or Perish, Berkeley, 1976.

[S1] T. Stanford, Finite-type invariants of knots, links, and graphs, Topology 35 (1996), 1027-1050.

[S2] T. Stanford, The functoriality of Vassiliev-type invariants of links, braids, and knotted graphs, J. Knot Theory Ramifications 3 (1994), 247-262.

[V] V. A. Vassiliev, Cohomology of knot spaces, Theory of Singularities and its Applications (V. I. Arnold, ed.), Advances in Soviet Mathematics, Vol. 1, Amer. Math. Soc., Providence, RI, 1990, pp. 23-69. 